\section{Cahiers de Narratologie}

Analyse et théorie narratives

$24 \mid 2013$

Avant-gardes et littérature narrative

\title{
Écriture manifestaire de Marinetti (1936-39)
}

Pour une littérature corporatiste coloniale autarcique et synthétique

Matteo D'Ambrosio

\section{(2) OpenEdition}

Journals

Édition électronique

URL : http://journals.openedition.org/narratologie/6731

DOI : $10.4000 /$ narratologie.6731

ISSN : 1765-307X

Éditeur

LIRCES

Référence électronique

Matteo D'Ambrosio, «Écriture manifestaire de Marinetti (1936-39)

», Cahiers de Narratologie [En ligne], 24 | 2013, mis en ligne le 18 septembre 2013, consulté le 30 avril

2019. URL : http://journals.openedition.org/narratologie/6731 ; DOI : 10.4000/narratologie.6731

Ce document a été généré automatiquement le 30 avril 2019

Article L.111-1 du Code de la propriété intellectuelle. 


\title{
Écriture manifestaire de Marinetti (1936-39)
}

Pour une littérature corporatiste coloniale autarcique et synthétique

\author{
Matteo D'Ambrosio
}

\section{NOTE DE L'ÉDITEUR}

(Traduit par Barbara Meazzi, revu par Isabelle Krzywkowski)

Dans la période considérée, marquée par de profonds changements dans la politique du régime fasciste (« africanisme » colonial, proclamation de l'Empire, autarcie, approche de la Seconde Guerre mondiale), Marinetti ne se consacre pas seulement et frénétiquement à la fortune du mouvement futuriste. Les documents publiés ici doivent principalement être reliés à son travail d'académicien, caractérisé par de nombreuses responsabilités institutionnelles. Il convient de rappeler quelques-unes de ces responsabilités : il était par exemple le directeur du Syndicat National «Autori e Scrittori » et de revues telles que Azione Imperiale; il présidait des jurys de prix - littéraires en particulier, mais pas seulement - et des institutions culturelles comme les «Stanze del Libro » à Rome ; il s'occupait de la diffusion de l'image de la nation, à la fois sur les plans politique et culturel, en prononçant des conférences en Italie et à l'étranger.

Les manifestes que nous reproduisons - qu'il conviendra sans doute d'analyser ailleurs de manière plus approfondie - font clairement apparaître l'indissociabilité entre l'illustration de la politique fasciste et les programmes du futurisme; du reste, le mouvement créé par Marinetti est à cette époque fortement engagé dans la modélisation des pratiques discursives de la propagande. Pendant cette période, le renouvellement dans les arts, que l'on voudrait continuer à garantir, n'est malheureusement reconnaissable ni dans les intentions exprimées, ni dans les résultats obtenus par les nouveaux manifestes : l'on s'attendrait à une toute autre teneur des manifestes consacrés par exemple au théâtre (1937) ou bien au roman (1939). 


\section{« Al Duce i ringraziamenti speciali ed entusiasti di noi poeti... » (mai 1936)}

Le 9 mai 1936 Mussolini, lors d'un discours, proclame la naissance de l'Empire. Au même moment est créée la revue mensuelle du Syndicat national des « Autori e Scrittori » : elle est dirigée par le secrétaire Corrado Govoni et par Marinetti, qui adresse un message à Mussolini depuis la première page du premier numéro ${ }^{1}$.

En août, une nouvelle revue est créée, Azione Imperiale, « recueil mensuel illustré de la création fasciste ${ }^{2}$ ", dirigée par Cesco Colagrosso et par Marinetti lui-même, qui signe le texte programmatique d'ouverture ${ }^{3}$. Marinetti désigne par "orientation des arts » l'« expression [...] de [...] la vie fasciste » et semble considérer la nouvelle orientation du régime comme la " préparation scientifique et pratique à la guerre » [pour le texte italien, $c f$. annexe 1]. Ce texte fournira les principaux arguments utilisés par l'écrivain catholique français Jules Romains pour attaquer à son tour le chef du mouvement futuriste, le 8 septembre 1936, lors du Congrès du Pen Club à Buenos Aires ${ }^{4}$. Marinetti répondra à cette attaque et aux attaques suivantes en 1940, depuis la première page de Autori e Scrittori ${ }^{5}$.

Dans le manifeste qu'il rédige pour répondre à une enquête lancée par la revue florentine La Scena illustrata ${ }^{6}$, Marinetti déclare souhaiter que la cuisine, en tant qu'art, devienne « Impériale », conformément aux transformations politiques italiennes.

\section{«Poesia e Arti Corporative » (8 mars 1937)}

Une année plus tard, l'Académicien Marinetti, adhérent au régime fasciste auquel, en tant que leader du futurisme, il assure et garantit la collaboration du mouvement sur le plan artistique, modifie les modalités de ses interventions : il prend position par rapport à la littérature et lance une nouvelle tendance qu'il appellera, plus tard, «La poésie des technicismes ${ }^{7} »$.

Dans le manifeste «Poesia e Arti Corporative », daté «Littoria, 8 mars 1937 » [annexe 2], mais probablement publié pour la première fois en avril ${ }^{8}$, il est signalé que plusieurs écrivains et poètes futuristes ont collaboré à sa rédaction ${ }^{9}$. La nouvelle typologie d'œuvres littéraires et artistiques évoquée - définie selon les principes de «transfiguration » et d' " exaltation »- montre l'assimilation opérée par le futurisme des projets politiques du régime, de sa presse et de ses hiérarchies. Étant donné les thématiques développées, cette action littéraire mériterait une place à part entière dans l'histoire des relations entre la littérature et l'industrie culturelle italiennes. Elle est confiée à la dernière génération des « jeunes puissants et équilibrés », qui « ... embrassent avec foi futuriste dans l'Empire créateur et avec inspiration certaine " "le technicisme mécanique chimique » et abandonnent, du côté de l'écriture, « la rhétorique superposées des verbalisations [...] de seconde main ${ }^{10} »$ :

Sans la désormais rance symbolique de la charrue de l'aigle de la faux de l'enclume du marteau abolie par les aéroplanes semeurs par les centrales électriques par les marteaux hydrauliques et les charrues à moteur nous voulons creuser directement chaque métier dans sa technique typique et dans sa productivité typique afin d'en extraire les frissons de la poésie ${ }^{11}$.

L'image du travail est alors soustraite à l'Italie rurale et aux données concernant le retard du système industriel, pour être insérée dans le contexte de la modernité technologique, 
dont le processus d'«idéalisation» vient opportunément d'être lancé. Le mouvement futuriste peut en effet se vanter de la pertinente production d'un assez grand nombre d'œuvres consacrées à la modernité technologique, qui ne sont pas seulement littéraires et sont parfois publiées dans des textes isolés ${ }^{12}$ :

Par groupes corporativement certains idéalisent chimie et industrie (Marinetti dans Poema della luce tessuta Folgore dans Sensazione fisica di materia Notari dans Romanzo d'un bilancio di podestà Buzzi dans Popolo canta così Farfa dans Tuberie e Tenerezze fresatorie Tullio d'Albisola dans Ceramiche Civello ${ }^{13}$ dans Aviazione) d'autres idéalisent commerces finance et agriculture (Azari dans Impiegato di banca Marinetti dans Poema del Porto di Rotterdam Marinetti Buzzi Govoni Masnata Scurto dans leurs poèmes sur Gli affari del Porto di Genova Scurto dans Poema della risaia Giardina dans Quand'ero pecoraio et Buccafusca dans Tecnica d'una cordata) d'autres idéalisent la technique de guerre (Marinetti dans Poema ${ }^{14}$ del Golfo della Spezia et dans Poema Africano della 28 Ottobre le maître Pratella dans L'Aviatore Dro le maître Giuntini dans Battaglia di terra mare cielo ${ }^{15}$ le maître Brizzi dans Gioia dei mitraglieri et Orgoglio dei chimici) Pino Masnata idéalise l'anatomie dans Poesia dei ferri chirurgici ${ }^{16}$.

Avant d'aborder directement le thème du travail, Marinetti dénonce le fait que, avec leur " sensibilité malheureuse », les poètes " passéistes » qui en ont fait l'éloge n'ont pas réussi à s'éloigner d'une «sensibilité d'âpreté fatigue ennui sacrifice voué à se réjouir pour le pot d'échappement du congé dominical ${ }^{17}$ ». À présent, il faut plutôt «aller au cœur des différentes techniques avec des outils adéquats inspirateurs chacun avec sa propre nomenclature à vivifier et avec sa sensibilité spécialisé18 ${ }^{18}$, non sans exclure " un certain nombre de travaux jugés prosaïques monotones gris donc dépourvus de poésie ${ }^{19} »$ : tous les efforts doivent être concentrés dans cette direction, en s'appuyant sur les principes désormais sédimentés du système idéologique futuriste.

Pour atteindre une efficace corporation poésie et art doivent magnifier chaque travail manifester les qualités suivantes : $1^{\circ}$ optimisme antinostalgique $2^{\circ}$ simplicité antirhétorique $3^{\circ}$ originalité $4^{\circ}$ variété $5^{\circ}$ intensité $6^{\circ}$ dynamisme $7^{\circ}$ synthèse $8^{\circ}$ tactilisme typique $9^{\circ}$ olfactisme typique $10^{\circ}$ bruitisme typique ${ }^{20}$.

Ailleurs émerge une référence aux expériences des artistes qui, suivant les lignes du projet de nouvelle littérature coloniale élaboré par Marinetti ${ }^{21}$ et approuvé par Mussolini, étaient en route pour l'Afrique orientale afin d'en "transfigurer» les caractères (y compris le paysage) d'un point de vue créateur :

Quant au désert qui signifie en réalité un vide aride et monotone il n'existe en dehors de cela meilleure preuve pour les poètes capables d'inventer un tourisme littéraire spécial enrichi de poésie fatigue ennuyeuse manque absolu de variété de couleurs et de formes désespérées mélancoliques sous les étoiles puanteur nauséabonde de bivouacs et tentes entre les chameaux absence de toilettes confortables présences des fientes manque de désaltérants dysenterie au guet engourdissement des membres ennui des conversations ennuyées par l'absolue répétition de rares émotions visuelles ${ }^{22}$.

Quoi qu'il en soit, l'on admet qu'il n'existe pas encore « de poésie de la joie concrète du travail personnellement raffiné23 ». Cela est démontré par «la récente anthologie Splendore della poesia italiana éditée par Corrado Govoni ${ }^{24}$ »" où, en examinant "les différents pourcentages des thèmes d'inspiration ", l'on découvre la prépondérance " de $80 \%$ d'amour platonique délibérément systématiquement malheureux désespéré déçu ${ }^{25}$ ». 


\section{« Contro il teatro morto. Contro il romanzone analitico. Contro il negrismo musicale » (22 octobre 1937)}

En octobre, Marinetti signe, avec Bruno Corra, revenu dans les rangs du futurisme pour l'occasion, le manifeste "Contro il teatro morto. Contro il romanzone analitico. Contro il negrismo musicale » [annexe ${ }^{26}$ ]. Il y réaffirme le principe de la «transfiguration », terme par lequel il indique généralement la fiction expressive et ses capacités à produire de " nouvelles réalités ». Il oppose l'écriture synthétique à l'écriture analytique : la première, caractérisée par l'originalité, l'invention et par un certain dynamisme, transfigure les choses suivant un «art de raconter à l'italienne souple synthétique et dynamique ${ }^{27}$ ». En l'absence de références explicites, les modèles devraient peut-être être identifiés, à partir de l'exemplum de Tacite $^{28}$, aux grands auteurs de la tradition nationale italienne, ceux-là mêmes auxquels Marinetti dédiait ses Commemorazioni in avanti ${ }^{29}$, surtout après sa nomination au titre d'Académicien d'Italie et en particulier entre 1936 et 1939. La référence à l'écriture en prose complète des considérations que Marinetti exprime à propos de la poésie dans un manifeste de 1934, "Errori dell'arte del tempo fascista », où il renvoie à $\mathrm{d}^{\prime}$ ' authentiques poètes modernes italiens dont le lyrisme est congénital, spontané, incontrôlable et irréductible ${ }^{30} »$ mais dont il évite de citer les noms. En vérité, le manifeste s'en prend plutôt et surtout au "théâtre mort en plein air ", à savoir les spectacles d'été qui ont instrumentalement exploité « Le projet du régime d'un "Théâtre pour les masses" [...] afin d'opérer un "retour à l'ancien" », le but étant de proposer des textes adaptés à un public ayant une "culture livresque " plutôt qu'au "peuple de la grande Italie fasciste ${ }^{31} »$. Partant de ses meilleurs manifestes de la fin de $1932^{32}$, Marinetti rappelle que le théâtre doit être «électromécanique» et profiter d'une «scène tournante ». La polémique à l'encontre des "amphithéâtres antiques » rappelle le manifeste contre le théâtre grec de Syracuse ${ }^{33}$, où il s'en prenait à Ettore Romagnoli. Ce dernier, malgré tout, était devenu dans les années trente un attentif et amical observateur du théâtre futuriste, et pour cela avait reçu des éloges à propos de ses « prodigieuses transformations de chefs-d'œuvre tels que l'œEdipe Roi ${ }^{34}$ ».

Un autre changement important à signaler concerne l'évaluation positive de l'influence du cinéma sur le théâtre, dont on avait beaucoup discuté dans les rangs futuristes. Marinetti s'en prend au "négrisme» du jazz, une nouvelle variante de l'antiaméricanisme, en associant à la fois son idiosyncrasie pour le tango, exprimée jadis dans un manifeste de $1914^{35}$, et l'exaltation de la tradition musicale populaire napolitaine, dont il est question à cette même époque dans le manifeste sur la déclamation futuriste (imprimé avec un retard de deux ans ${ }^{36}$ ).

Dans le deuxième paragraphe du manifeste, on peut lire l'introduction polémique du manifeste sur le roman « synthétique » :

Nous les transfigurateurs les créateurs des nouvelles réalités amis de la synthèse à la Tacite nous condamnons le gros roman analytique qui ignorant l'indispensable originalité de conception l'indispensable invention de types et l'indispensable choix de mots et de gestes suggestifs dissèque avec pédanterie la vie quelconque d'un imbécile quelconque dans un environnement rendu quelconque par le manque de génie sélectionneur.

Pour obtenir l'adhésion de certains lecteurs avarement inquiets d'avoir beaucoup à lire en échange de leur argent les romanciers subissent le gris froid couvercle de nuages qui depuis le nord descend favorable à un dépouillage de remords et de 
commérages de mendiant routier et ils suffoquent sous une psychologie abrutissante à long métrage notre art svelte synthétique et dynamique de raconter à l'italienne.

Que ces imitateurs et ces exagérateurs du monologue intérieur et des blagues-àpart des vieilles comédies avec longs vomissements freudiens de banalités soient relégués dans la pharmacologie comme de bons somnifères à prescrire avec prudence ${ }^{37}$.

\section{« Ampliare l'ispirazione letteraria cantando l'Etiopia, $i$ telai di Biella e $i$ vini italiani etc. » (novembre 1937)}

Seulement un mois plus tard, c'est le tour du manifeste «Ampliare l'ispirazione letteraria cantando l'Etiopia, i telai di Biella e i vini italiani etc. » [annexe $4^{38}$. La référence à l'industrie textile reprend certains éléments déjà insérés dans « Poesia e Arti Corporative »; le renvoi à la partie sur les vins intègre en revanche le contenu du tract «Invito ai poeti ${ }^{39}$ ", lancé quelques mois auparavant lors d'une manifestation qui avait échouét ${ }^{40}$. Une fois de plus, le discours sur la littérature coloniale revient: Marinetti l'avait lancé une première fois dans le manifeste "Invito alla guerra africana ${ }^{41}$ ", adressé " aux écrivains et aux artistes d'Italie » et il l'avait repris trois ans plus tard, dans une version élargie intitulée "L'Africa generatrice e ispiratrice di poesia e arti » [annexe $5^{42}$ ].

L'adhésion au projet colonial du régime avait assuré la participation du mouvement futuriste dans différents domaines artistiques, à commencer par les arts visuels, puis lors des deux premières expositions d'art colonial ${ }^{43}$ et de l'exposition du livre colonial fasciste (Rome, avril 1936). Une place sera fait à l'«africanisme» pictural futuriste encore pendant les premières années de la Seconde Guerre mondiale, et notamment lors de l'exposition napolitaine des Terres d'Outre-mer de 1941, et du manifeste rédigé pour l'occasion ${ }^{44}$.

En plus de ces "commémorations", Marinetti avait l'habitude de participer à toutes sortes de remises de prix, auxquels il a consacré une bonne partie de son manifeste " Ampliare l'ispirazione letteraria». Le 24 juin 1939, par exemple, il est occupé à justifier l'attribution du prix Firenze au livre Cleopatra contro Roma $a^{45}$ de Maffio Maffii, directeur du journal quotidien florentin La Nazione. Dans ce cas, assez rare, les deux modes d'intervention critique utilisés par Marinetti, à savoir la «misurazione ${ }^{46}$ " et les " commemorazioni in avanti » sont remplacées par les formes de l'éloge, Maffii et son livre devant être célébrés d'abord parce que l'auteur a réussi à se débarrasser de «l'ancien problème philosophique et littéraire », de la soi-disant " vérité historique » qui, « n'étant pas accessible de manière absolue, doit être rejetée ». Marinetti se rend compte que, tout compte fait, la narration se caractérise toujours par rapport à une "vérité très relative ". Sensible au culte du licteur romain, Marinetti loue enfin l'« orgueil patriotique » exprimé par Maffii, sans recourir à l'« histoire fictive ", tout en soulignant le " prestige de Rome ${ }^{47}$ $»$ et de la civilisation latine.

\section{« Autarchia letteraria » (décembre 1937)}

En décembre 1937, avec le manifeste de l'« autarcie littéraire » signé également par le fidèle Govoni, Marinetti prend part au débat en cours sur la publication des traductions de livres étrangers. Il demande que le nombre de traductions diminue quantitativement 
et qu'on se limite aux " authentiques chefs-d'œuvre », de manière à exclure des œuvres comme Sous le regard des étoiles d'A. J. Cronin :

Nous considérons comme indispensable à la spiritualité italienne fasciste mussolinienne la traduction et la publication en Italie d'authentiques chefsd'œuvre de toute la littérature étrangère.

Nous considérons également comme indispensable à l'autarcie littéraire qu'il est désormais urgent de rejeter la traduction et la publication des trois quarts des œuvres étrangères que certains éditeurs imposent en se basant sur le vice italien ancien et permanent et insuffisamment vilipendé que nous appelons xénophilie.

Cette xénophilie ayant pour conséquence le dénigrement de la production littéraire italienne trouve son ignoble nourriture dans la multiplication de romans très médiocres.

Nous proposons ainsi que la traduction et la publication d'œuvres étrangères soit a priori réduite de moitié et strictement réglementée.

Nous sommes prêts à prouver livre à la main la pudibonde stupidité pessimiste et les minutieuses idioties défaitistes ou anti-italiennes qui caractérisent des livres étrangers tels que Sous le regard des étoiles ${ }^{48}$.

La préoccupation du régime de discipliner "rigoureusement " tous les aspects socioculturels est redéfinie en admettant la diffusion de la « xénophilie », l'un des fleurons de Marinetti, qui l'avait stigmatisée au moins dès son manifeste « Contro l'esterofilia » de 1931

49. Les interventions de Marinetti sur les auteurs étrangers sont par conséquent plutôt rares, exception faite, à cette époque, pour l'écrivain catholique Paul Claudel, présenté comme « le plus grand poète de la France contemporaine ${ }^{50}$ ».

La défense de la culture italienne face aux ingérences étrangères passe à la fois par une prise de position par rapport aux traductions ${ }^{51}$ et par des projets de diffusion du livre. Marinetti avait imaginé la création de bibliothèques populaires et la mise en place d'un " camion remorque du livre », capable d'atteindre les lieus les plus isolés ${ }^{52}$. Pendant l'été 1938, le Ministère de la Culture Populaire pourra affronter « le problème de l'assainissement libraire ${ }^{53}$ ». Ennemi juré de la "xénophilie ", comme nous l'avons déjà relevé, Marinetti essayait de la contrebalancer en utilisant massivement les néologismes (comme dans le « manifeste de la cuisine futuriste » de 193054). Il n'est par conséquent pas étonnant de le retrouver dans la commission restreinte chargée par l'Istituto dell'Enciclopedia italiana de réviser les termes de dérivation étrangère afin de les remplacer par des néologismes italiens adéquats. Adelmo Cicogna consacre un livre à l' Autarchia della lingu $a^{55}$, dont Marinetti rédige la préface où, entre autres, on peut lire :

Le Premier problème autarcique est l'italianité de la langue. [...] Il faut cesser d'utiliser des mots étrangers [...] nous voulons ôter tous les mots étrangers de la langue italienne ${ }^{56}[\ldots]$.

\section{«L'Africa generatrice e ispiratrice di Poesia e Arti » (octobre 1938)}

Invité à intervenir au congrès « Volta » qui se tient à Rome en octobre 1938, Marinetti se penche à nouveau sur la littérature coloniale et publie le manifeste «L'Africa generatrice $e$ ispiratrice di Poesia e Arti " [annexe 6], en mettant en exergue les œuvres des auteurs non italiens et non futuristes. Dans la liste, cependant, il insère cinq de ses livres :

L'on distingue en littérature les œuvres de Ferdinando Martini (Africa Italiana)

Edmondo De Amicis (Marocco) F. T. Marinetti (Mafarka il futurista - Gli Indomabili - Il

Tamburo di fuoco - Il fascino dell'Egitto - Il Poema [africano] della Divisione 28 Ottobre) 
Domenico Tumiati (Tripolitania ${ }^{57}$ ) Guelfo Civinini (Abissinia) Appelius Milanesi Bruno Corra Victor Hugo Gerard [sic] de Nerval Baudelaire Flaubert Loti Jean Lorrain André Gide Paul Morand Randau ${ }^{58}$.

Cette juxtaposition d'œuvres d'auteurs étrangers au futurisme dans un manifeste pourrait paraître surprenante. Il convient de rappeler, toutefois, que Marinetti en 1928 avait promu, présidé et rédigé le programme des Dix, un groupe d'auteurs qui avaient écrit, de manière anonyme, les différents chapitres d'un roman intitulé Lo Zar non è morto 59. L'initiative avait été désapprouvée par certains futuristes de la première heure comme Settimelli ${ }^{60}$, qui n'en partageait ni les justifications littéraires ni la stratégie.

\section{« Manifesto del Trentennale del Futurismo » (mai 1940); « Manifesto degli aeropoeti nel trentennale del futurismo " (septembre 1939)}

En février 1939, le trentième anniversaire du mouvement futuriste est célébré tout en fait en sourdine. L'anniversaire ne donna lieu à aucune intervention significative sur le versant de la réflexion critique; même sur le plan artistique, aucun artiste futuriste ne proposa pour l'occasion d'œuvre particulièrement significative. Le jour de l'anniversaire, les futuristes se rencontrèrent au Lido di Roma et dans les salles de la Quadriennale. Marinetti se limita à proposer une « Formula letteraria artistica per la creazione delle opere future » [Formule littéraire artistique pour la création d'ouvrages futuristes], diffusée dans un tract par l'Agence « A. L. A.» [annexe 7-161].

Le diagramme où sont opposées l'écriture analytique et l'écriture synthétique dont il a été question auparavant réapparaît dans le «Manifesto degli aeropoeti nel trentennale del futurismo » [annexe 7-2]. Une fois de plus, Marinetti s'insurge contre le

Gros roman analytique socialo-pessimiste à tendance communiste (Thomas Mann Jules Romains) dégénération du «monologue intérieur » de Dujardin ${ }^{62}$ où Proust et Joyce diluent nos mots en liberté synthétiques dynamiques simultanés dans une diarrhée de paroles ${ }^{63}$.

Ce texte reprend un article de 1934, dans lequel le «monologue intérieur» est défini comme " une sincérité absolue alogique de conscience et du sub-conscient ». Joyce, selon Marinetti, se serait inspiré, outre de Les Lauriers sont coupés d'Édouard Dujardin (1887), des « lyriques motslibristes » : « certaines [...] et tout spécialement Dunes, contiennent aussi le soi-disant "monologue intérieur ${ }^{64 "}$ ".

La datation attribuée au texte de référence, paru jadis dans Lacerba ${ }^{65}$ en italien, est plutôt intéressante : le titre, cité en français, laisse penser à une publication antérieure ${ }^{66}$, ce qui fait que le poème résulterait finalement d'une anticipation des manifestes du motlibrisme, dont le premier est de $1912^{67}$.

Les trois premiers points seront inscrits à l'intérieur de « Un messaggio del poeta Marinetti ai poeti artisti futuristi di A.O.I. », imprimés sur un tract de l'Agence «A. L. A. » le 24 mai $1939^{68}$, et repris par la presse dans les mois suivants. Le deuxième point présente une nouvelle version de l'extrait déjà cité :

2) Gros roman analytique social-pessimiste à tendance communiste (Thomas Mann Jules Romains) type modéré du monologue intérieur de Dujardin ${ }^{69}$ que Proust et Joyce corrompant nos mots en liberté essentiels simultanés dans toutes leurs paroles vaines apportèrent un vomissement psychologique à répétition ${ }^{70}$. 


\section{« Il romanzo sintetico » (24 décembre 1939)}

À la fin de 1939, Marinetti lance le manifeste «Il romanzo sintetico ${ }^{71}$ » [annexe 8], considéré de nos jours comme une démonstration du fait que, même dans ce domaine, comme dans le cinéma et dans la photographie, le futurisme n'a pas fourni d'innovations importantes et significatives. Il s'agit en fait d'un manifeste tardif et qui n'aura pas de suite. Le manifeste de Marinetti paraît en même temps que Picchiata nell'amore de Piero Bellanova ${ }^{72}$ , dont le fondateur du futurisme, dans sa courte préface, célèbre la «spontanéité », le trouvant « étonnant et hilarant », « inattendu et surprenant... ».

On a écrit à juste titre que ce manifeste est «anachronique, inutile et pompeux [...] mettant surtout l'accent sur l'écart entre les prétentions révolutionnaires et la réalité conservatrice, ce qui accentue la saveur d'un retour à l'ordre et de la tradition ${ }^{73}$ ". Discutable, en revanche, l'idée selon laquelle la littérature narrative serait « un genre marginal dans la production littéraire du mouvement ${ }^{74}$ » et que les futuristes n'auraient montré «aucun enthousiasme pour le roman, et très peu d'intérêt pour un genre considéré dépassé et vieilli [...] obsolète et dépourvu d'intérêt ${ }^{75} \%$. Le corpus hétérogène de la littérature narrative futuriste, quantitativement important, ne relève certainement pas d'un " genre négligé ${ }^{76}$ ", malgré les limites qualitatives d'une prose qui demeure d'un symbolisme tardif, comme cela se fit ailleurs en Italie (c'est le cas de Sentimento del tempo de Giuseppe Ungaretti, paru en 1933). Les cas d'adhésion aux formes discursives de la littérature populaire sont tout aussi nombreux (d'autant que, comme Marinetti l'écrit dans le manifeste, " en général le public ne fait pas la différence ») : ici, il n'est pas rare d'assister à la superposition d'images symbolistes avec l'occultisme, le paranormal ${ }^{77}$, en récupérant ainsi certaines suggestions post-dannunziennes. À cela il faut ajouter le fait que la précoce normalisation syntaxique et formelle a sans doute nui aux résultats atteints par la littérature narrative futuriste - et à leur évaluation.

En vérité, au sein du mouvement futuriste, l'intérêt pour la prose n'a jamais manqué : le manifeste du roman synthétique peut être considéré comme un aveu à la fois de l'échec des tentatives de conquérir un nouveau « sommet » (en Italie et au sein des avant-gardes, et par rapport à la littérature étrangère) et, indirectement, des limites des œuvres futuristes citées. Il est intéressant que, parmi les grands auteurs nommés, apparaisse aussi Giovanni Verga, que peu de temps auparavant Marinetti avait défini «assez futuriste ${ }^{78} »$.

Outre l'absence d'œuvres narratives montrant que les indications de ce manifeste avaient bien été mises en pratique, l'actuelle sous-estimation de la littérature narrative futuriste a au fond été favorisée par Marinetti lui-même : avec insistance, en effet, il a défini la littérature narrative futuriste comme le développement cohérent du motlibrisme qu'il considérait non pas comme un nouveau code poétique, mais comme une typologie d'écriture poétisée. Voilà pourquoi on a pu affirmer que « les formes verbales futuristes [...] semblent incompatibles avec les exigences du roman ${ }^{79} »$. Dans un écrit de 1921, Marinetti déclare en effet que Lo stile narrativo " naît du motlibrisme ${ }^{80}$ " : cette affirmation sera reprise d'abord dans l'introduction aux Indomabili ${ }^{81}$ puis ailleurs ${ }^{82}$, avec quelques variantes. Dans la première partie du texte, Marinetti se contente d'opposer un article d'Ugo Ojetti (qui considère le futurisme comme une ruine passéiste plagiée par Dada) à certains commentaires positifs ${ }^{83}$, qui confirment le recours désormais habituel et contradictoire au principe d'autorité. Dans la deuxième partie, il cite, à titre d'exemples, 
deux textes, l'un extrait de Crepapelle de Folgore et l'autre d'un roman de 1920 d'Angelo Rognoni, La veste che faceva frou frou ${ }^{84}$, auxquels il joint un de ses écrits sur la lecture basée sur la « simultanéité des rythmes ${ }^{85}$ ».

Ces difficultés historiographiques persistantes concernent surtout la vérification de l'application des principes de l'esthétique futuriste et, en particulier, des théories de Marinetti sur l'écriture, largement redimensionnées par certaines de ses œuvres telles que Gli Indomabili, complètement dépourvues d'une vocation expérimentale identifiable ${ }^{86}$, ou bien Originalità russa di masse distanze radio-cuori ${ }^{87}$ ou, encore, Venezianella e Studentaccio, récemment publié pour la première fois ${ }^{88}$. En l'absence d'autres manifestes consacrés à la littérature narrative, il faudra que les futures reconstructions historiographiques tiennent compte des textes et des transcriptions des conférences de Marinetti (comme celle de Trieste, en $1929^{89}$ ), de ses interventions consacrées aux œuvres d'autrui (introductions, préfaces, «collaudi»), ainsi que de ses rares écrits à propos des ouvrages individuels des auteurs futuristes ${ }^{90}$. Il n'existe pas encore de bibliographie fiable de ces contributions, mais il n'est pas difficile de prévoir que de nombreux textes écrits à l'occasion d'événements particuliers s'avèreront souvent élaborés dans l'approximation, ou bien inspirés polémiquement par la nécessité stratégique de s'opposer au succès de certains auteurs étrangers. Une analyse textuelle approfondie pourrait également permettre d'appréhender autrement le terme «synthétique » utilisé dès 1917 dans Sam Dunn è morto de Corra ${ }^{91}$ : la critique en a souligné les valeurs expressives, mais il conviendra d'élargir l'analyse à d'autres textes en prose.

Comme pour le trentième anniversaire, les invitations réitérées à la diversification sont une preuve de l'insatisfaction de Marinetti face aux résultats obtenus par les futuristes par rapport à la recherche de nouveaux arts et de nouveaux types d'écriture, et ce pas seulement pour répondre à des besoins de renouvellement créateur : cela confirmerait d'ailleurs la vocation propre à l'avant-garde d'être un laboratoire expérimental. Cela dit, la politique culturelle fasciste va encourager de plus en plus les écrivains futuristes à s'orienter vers des pratiques littéraires accessibles aux masses, vers, en somme, une littérature populaire ${ }^{92}$, suivant ce qui s'était déjà produit pour le théâtre, le but étant de créer des formes propagandistes d'agrégation par le biais desquelles le régime souhaitait encadrer et cadrer la société italienne. À ce propos, il est tout à fait légitime de se demander lesquelles, parmi les nombreuses œuvres futuristes en prose, peuvent réellement être considérées comme de la littérature d'avant-garde.

Le lancement du manifeste sur le roman « synthétique » a été suivi par deux enquêtes. La première fut promue par l'Agence "A. L. A. », et parmi les personnes interviewées on trouve l'académicien Umberto Giordano. L'« Inchiesta sul romanzo sintetico futurista » était centrée sur deux questions : «1) Approuvez-vous le Manifeste Futuriste du roman synthétique ? 2) Êtes-vous pour ou contre ce manifeste? ». L'enquête avait été lancée par la revue romaine Il romanzo per tutti qui, auparavant, dans le dossier numéro 6, avait publié le manifeste ${ }^{93}$.

Le leader du futurisme reviendra sur le sujet à l'occasion d'une publication du futuriste calabrais Geppo Tedeschi, dont le «collaudo » est en grande partie une revue de presse de jugements exprimés sur le sujet et de messages d'éloges signés non seulement par les camarades futuristes, mais aussi par un général ${ }^{94}$. 


\section{« Manifesto futurista per la formazione dell'ideale scrittore cattolico » (mai 1941)}

Dans une lettre datée du 14 novembre 1939, Marinetti avait été invité par Cesare Angelini, néo-recteur du Collegio Borromeo de Pavie, à prononcer une conférence sur les trente ans d'activité du mouvement ${ }^{95}$. Par la suite, le 20 mai 1941, le futur auteur du Aeropoema di Gesù ${ }^{96}$ consacre une conférence, accueillie dans les Stanze del Libro de Rome, à un livre écrit par Angelini, Invito in Terrasanta97, d'où il extrait les quinze principes développés dans le «Manifesto futurista per la formazione dell'ideale scrittore cattolico" [annexe 998]. Certains de ces principes qui, dans le « manifeste du Tactilisme ${ }^{99}$ » étaient posés dans un contexte laïque (comme l'Amour, avec une majuscule, redéfini comme un sentiment de l'émotivité), prennent désormais une coloration religieuse. Du reste, le promoteur de la "dévaticanisation » avait gardé le silence lorsque, après le Concordat de 1929 et la prise de position conséquente du régime par rapport à la religion catholique, certains artistes avant-gardistes - y compris certains futuristes comme Emilio Settimelli ${ }^{100}$ - en avaient revendiqué l'actualité. À présent, Marinetti met en exergue certains contenus qui devraient caractériser une écriture confessionnelle au nom de la foi «absolue » et « dépourvue de logique » : il souhaiterait voir émerger dans cette écriture, toutefois, un minimum de motlibrisme, «des adjectifs imprévisibles» et moins de signes de ponctuation.

\section{NOTES}

1. «Al Duce i ringraziamenti speciali ed entusiasti di noi poeti per aver dotato le nostre ispirazioni d'una nuova vita guerriera africana imperiale straricca d'avventure e pericoli popoli da spiritualizzare faune flore mèssi miniere d'oro platino ferro carbone petrolio solitudini gonfie di divino morti eroiche per la bellezza immensificata della sempre più adorata Italia » [Au Duce nos remerciements spéciaux de poètes pour avoir pourvu nos inspirations d'une nouvelle vie guerrière africaine impériale très riche en aventures et dangers peuples à spiritualiser faunes flores récoltes mines d'or platine fer charbon pétrole solitudes gonflées de divin morts héroïques pour la beauté immensifiée par l'Italie de plus en plus adorée], Autori e Scrittori, Roma, I, n. 1, mai 1936, p. 1.

2. « rassegna mensile illustrata della creazione fascista».

3. Azione Imperiale, Roma, I, n. 1, août 1936, p. 1.

4. Pendant son séjour en Argentine, Marinetti rencontra à plusieurs reprises la communauté des immigrés italiens. Il défendit l'impérialisme mussolinien et critiqua l'embargo imposé à l'Italie par la communauté internationale suite à l'invasion de l'Éthiopie. Le deuxième numéro de Autori e Scrittori s'ouvre sur « Il messaggio del Duce agli italiani dell'Argentina recato da Marinetti ».

5. «Risposta del poeta Marinetti agli scrittori Jules Romains, Duhamel, Pierard, Benjamin Cremieux, Ludwig, Wolf, Wells », Autori e Scrittori, Roma, V, n. 7, juillet 1940, p. 1.

6. F. T. Marinetti Accademico d'Italia, "Verso una Imperiale Arte Cucinaria», La Scena illustrata, Firenze, LIII, n. 5, mai 1938. 
7. Le manifeste « Poesia e Arti Corporative » sera en effet proposé à nouveau, sous le titre « La Poesia dei Tecnicismi » (voir la note suivante).

8. F. T. Marinetti, «Poesia e Arti Corporative. Manifesto futurista ", La Gazzetta del Popolo, Torino, 10 avril 1937, p. 3. Repris dans Franca Maria Corneli, L'aeropoema futurista dell'Umbria. Parole in libertà applaudite nel $1^{\circ}$ Dinamismo di Poesie guerriere (de Franca Maria Corneli Dina Cucini Maria Goretti Liguori Monelli Farfa Acquaviva) del Sindacato Autori Scrittori a Roma 8 maggio 1943-XXI. Con un saggio di Marinetti sulla Poesia dei tecnicismi, Roma, Edizioni futuriste di «Poesia » della Galleria nazionale d'arte futurista e aeropittura di guerra, 1943, p. 3-7 (sous le titre «La Poesia dei Tecnicismi» daté du 29 mai 1943; signé «F. T. Marinetti Sansepolcrista Accademico d'Italia $1^{\circ}$ Seniore della "23 Marzo" " et précédé par le renvoi à d'autres textes futuristes à inclure parmi les chefs-d'œuvre de la Poésie des Technicismes: "Il poema non umano dei tecnicismi di Marinetti La Poesia dei ferri chirurgici di Pino Masnata Il Poema della Rotativa $4^{\underline{a}}$ edizione di Guglielmo Jannelli suscitano polemiche acutizzate in ceffoni e cazzotti futuristi come quelli di Piazza De Ferrari a Genova pro il Poema del tecnicismo del Sulcis dell'ingegnere aeropoeta Eugenio Caracciolo e pro il Poema del Sottobosco di Alfonso Bossetti del gruppo Futurista Modenese Boccioni » II poema non umano dei tecnicismi de Marinetti La Poesia dei ferri chirurgici de Pino Masnata le Poema della Rotativa $4^{\mathrm{e}}$ édition de Guglielmo Jannelli suscitent polémiques aiguisées en gifles et coups de poing futuristes comme ceux de la Piazza De Ferrari à Gênes en soutien au Poema del tecnicismo del Sulcis de l'ingénieur aéropoète Eugenio Caracciolo et en soutien au Poema del Sottobosco d'Alfonso Bossetti du groupe Futurista Modenese Boccioni].

9. « BENEDETTA BRIZZI BUCCAFUSCA CARTA Tullio D’ALBISOLA DI GESE FARFA Pino MASNATA PRAMPOLINI SANZIN SIBÒ Luigi SCRIVO Ignazio SCURTO TEDESCHI TRECCA ».

10. "ventenni potenti ed equilibrati "; «abbracciano [...] con fede futurista nell'Impero creatore e con sicura ispirazione »; «il tecnicismo meccanico chimico »; « la sovrapposta retorica delle verbalizzazioni [...] usate ».

11. «Senza l'ormai rancida simbologia dell'aratro dell'aquila della falce dell'incudine del martello abolita dagli aeroplani seminatori dalle centrali elettriche dai magli idraulici e dalle motoaratrici vogliamo direttamente scavare ogni lavoro nella sua tipica tecnica e nella sua tipica produttività per estrarne $i$ brividi della poesia. »

12. Comme Tecnica d'una cordata d'E. Buccafusca, qu'il faut lire de bas en haut, publié dans Prima Linea, Napoli, I, n. 1, 25 mai 1935, p. 3 (sous le titre « Cordata»).

13. Le titre a été corrigé justement par «Aria madre» dans la version suivante, publiée dans F. T. Marinetti, Il Poema non umano dei tecnicismi, Milano, Arnoldo Mondadori, 1940 (sous le titre « Estrazione sistematica di nuovi splendori e nuove musiche dai tecnicismi »).

14. Corrigé « Aeropoema » dans Franca Maria Corneli, op. cit., p. 5.

15. Cette expression revient dans la réimpression du texte de la conférence de Marinetti à l'Académie d'Italie du 8 avril 1942 (imprimé sous forme d'opuscule intitulé La poesia sublime dell'esercito italiano), reproposé dans Mediterraneo futurista comme « Eroi italiani di terra mare e cielo» (Mediterraneo futurista, Roma, V, n.13, juin 1942, p.1-2; signé F.T. Marinetti Sansepolcrista).

16. «A gruppi corporativamente alcuni idealizzano chimica e industria (Marinetti nel Poema della luce tessuta Folgore in Sensazione fisica di materia Notari nel Romanzo d'un bilancio di podestà Buzzi in Popolo canta così Farfa in Tuberie e Tenerezze fresatorie Tullio d'Albisola in Ceramiche Civello in Aviazione) altri idealizzano commerci finanza e agricoltura (Azari nell'Impiegato di banca Marinetti nel Poema del Porto di Rotterdam Marinetti Buzzi Govoni Masnata Scurto nei poemi su Gli affari del Porto di Genova Scurto nel Poema della risaia Giardina in Quand'ero pecoraio e Buccafusca in Tecnica d'una cordata) altri idealizzano la tecnica di guerra (Marinetti nel Poema del Golfo della Spezia e nel Poema Africano della 28 Ottobre il maestro Pratella nell'Aviatore Dro il maestro Giuntini in Battaglia di terra mare cielo il maestro Brizzi nella Gioia dei mitraglieri e Orgoglio dei chimici) Pino Masnata idealizza l'anatomia nella Poesia dei ferri chirurgici. » 
17. « una sensibilità di asprezza fatica noia sacrificio teso a rallegrarsi per il tubo di scappamento della vacanza domenicale »

18. "entrare nel vivo delle tecniche diverse con i relativi utensili ispiratori ognuno con la sua nomenclatura da vivificare e con la relativa sensibilità specializzata »

19. " un certo numero di lavori giudicati prosaici monotoni grigi quindi privi di poesia »

20. "Per raggiungere un'efficacia corporativa poesia e arte devono magnificare ogni singolo lavoro manifestare le seguenti qualità: $1^{\circ}$ ottimismo antinostalgico $2^{\circ}$ semplicità antiretorica $3^{\circ}$ originalità $4^{\circ}$ varietà $5^{\circ}$ intensità $6^{\circ}$ dinamismo $7^{\circ}$ sintesi $8^{\circ}$ tipico tattilismo $9^{\circ}$ tipico olfattismo $10^{\circ}$ tipico rumorismo. » 21. $C f$. les informations fournies dans le dernier paragraphe du manifeste «L'Africa generatrice $e$ ispiratrice di poesia e arti » [annexe 6].

22. «In quanto al deserto che significa in realtà un vuoto arido e monotono non esiste fuori di esso maggiore prova per $i$ poeti che seppero inventare uno speciale turismo letterario arricchendo di poesia fatiche tediose assoluta mancanza di varietà di colori e di forme disperate malinconie sotto le stelle puzzo nauseante di bivacchi e tende fra i cammelli assenza di comode ritirate presenza degli sterchi mancanza di dissetanti dissenteria in agguato indolenzimento degli arti noia di conversazioni annoiate dalla assoluta ripetizione delle poche emozioni visive. "

23. "non esiste poesia della gioia concreta del lavoro personalmente raffinato "

24. Splendore della Poesia Italiana: dalle origini ai giorni nostri, Corrado Govoni (dir.), Milano, Hoepli, 1937.

25. "l'antologia recentissima Splendore della Poesia Italiana a cura di Corrado Govoni »; "le diverse percentuali dei motivi ispiratori"; "l'80\% di amore platonico volutamente sistematicamente infelice disperato deluso ".

26. F. T. Marinetti, Bruno Corra, «Contro il teatro morto. Contro il romanzone analitico. Contro il negrismo musicale. Manifesto futurista », La Gazzetta del Popolo, Torino, 22 octobre 1937, p. 3.

27. « snella sintetica e dinamica arte di narrare all'italiana »

28. Marinetti avait traduit, en 1928 , De situ et origine germanorum de Tacite, publié sous le titre La Germania (Milano, Istituto Editoriale Italiano ; édition bilingue).

29. Cf. Matteo D'Ambrosio, Le “Commemorazioni in avanti" di F. T. Marinetti. Futurismo e critica letteraria, Napoli, Liguori editore, 1999.

30. F. T. Marinetti dell'Accademia d'Italia, « Errori dell'arte del tempo fascista », Noi, Roma, II, n. 10, 16 juin 1934, p. 6.

31. "Il progetto di regime di un "Teatro di masse" [...] per operare un "ritorno all'antico" "; " preparazione libresca »; " popolaresca grande Italia fascista »

32. F. T. Marinetti, «Il teatro totale e la sua architettura " [1932], Teatro contemporaneo, Roma, V, n. 9, febbraio-maggio 1985, p. 377-384. Avec une présentation intitulée « Un inedito di Marinetti », signé Luce Marinetti et Mario Verdone (p. 373-375) ; «Il teatro totale di F. T. MARINETTI », in Almanacco Letterario Bompiani, Milano, 1933, p. 305-309.

33. «PER I FUTURISTI SICILIANI : Jannelli, Nicastro, Vann'Antò, Carrozza, Raciti, MANIFESTO FUTURISTA per le Rappresentazioni Classiche al Teatro Greco di Siracusa », [Siracusa], 16 avril 1921. Tract.

34. « prodigiose trasfigurazioni di capolavori come l'Edipo Re »

35. F. T. Marinetti, «Abbasso il Tango e Parsifal! Lettera futurista ad alcune amiche cosmopolite che dànno dei thè-tango e si parsifalizzano ", 11 janvier 1914; Milano, Direzione del Movimento Futurista. Tract.

36. F. T. Marinetti, "La Declamazione Dinamica e Sinottica. Manifesto futurista ", Milano, 11 mars 1916. Milano, Direzione del Movimento Futurista; in Francesco Cangiullo, Piedigrotta, Milano, Poesia, 1916, p. a-e.

37. « Noi trasfiguratori e creatori di nuove realtà amici della sintesi tacitiana condanniamo il romanzone analitico che ignorando la indispensabile originalità di concezione l'indispensabile invenzione di tipi e l'indispensabile scelta di parole e gesti suggestivi anatomizza pedantescamente la vita qualsiasi di qualsiasi 
fesso in un ambiente reso qualsiasi dalla mancanza d'ingegno selezionatore. Per farsi acquistare da certi lettori avaramente preoccupati di avere molto da leggere in compenso del loro denaro i romanzieri subiscono il grigio freddo coperchio di nuvole che dal nord scende favorevole ad uno spidocchiamento di rimorsi e pettegolezzi da mendicante stradale e soffocano sotto una abbrutente psicologia a lungo metraggio la nostra snella sintetica e dinamica arte di narrare all'italiana. Questi imitatori ed esageratori del monologo interiore e delle battute a - parte - delle vecchie commedie con lunghi vomiti freudiani di banalità siano relegati nella farmacologia come sonniferi sicuri da prescrivere con cautela. » Le manuscrit de huit feuillets est conservé à la Beinecke Rare Book \& Manuscript Library, Yale University. Il a probablement été imprimé.

38. F. T. Marinetti, «Ampliare l'ispirazione letteraria cantando l'Etiopia, i telai di Biella e i vini italiani etc. ", Autori e Scrittori, Roma, II, n. 11, novembre 1937, p. 1-2.

39. I poeti vignaioli F. T. Marinetti Corrado Govoni Giuseppe Lipparini, «Invito ai poeti », s.l., s.d.. Tract ; ensuite publié dans Autori e Scrittori, Roma, II, n. 8/9, août-septembre 1937, p. 9.

40. Deux ans plus tard seulement, le Prix «au poète original » sera décerné ex aequo à Pino Masnata, Dina Cucini et Maria Goretti. La cérémonie de remise du prix se déroula dans le parc de la Varignana, près de Castel San Pietro ( $c f$. «Pino Masnata Dina Cucini e Maria Goretti vincitori del Premio "Al Poeta Originale" e proclamati "Poeti Originali" ", Autori e Scrittori, Roma, IV, n.11, novembre 1939, p. 9). À cause de la guerre, le Prix sera par la suite suspendu.

41. F. T. Marinetti, "Invito alla guerra africana. Manifesto agli scrittori e agli artisti d'Italia ", La Gazzetta del Popolo, Torino, 30 juillet 1935, p. 3.

42. F. T. Marinetti, «L'Africa generatrice e ispiratrice di poesia e arti », Autori e Scrittori, Roma, III, n. 10, octobre 1938, p. 3-4.

43. Il mostra internazionale d'arte coloniale (Roma, octobre-décembre 1931) et II mostra internazionale d'arte coloniale (Napoli, octobre 1934-janvier 1935) ; cf. les catalogues respectifs, in Nuovi Archivi del Futurismo, I. Cataloghi di esposizioni, Enrico Crispolti (dir.), Roma, De Luca editori d'arte, 2010, p. 455-456 et 587-588.

44. Cf. le manifeste de Marinetti «Aeropitture africaniste degli aeropittori futuristi », in Ugo Ortona, Le Terre d'Oltremare e l'arte italiana contemporanea, Napoli, Edizioni della Mostra d'Oltremare, 1941, p. 234-235, republié pour la première fois in Matteo D'Ambrosio, Futurismo a Napoli. Indagini e documenti, Napoli, Liguori editore, 1995, p. 234-235 ; maintenant in Nuovi Archivi del Futurismo, I. Cataloghi di esposizioni, cit., p. 729.

45. Firenze, Vallecchi, 1939 ; après la guerre, il sera réimprimé à plusieurs reprises.

46. Cf. Matteo D'Ambrosio, "La critica non è mai esistita e non esiste". A proposito della misurazione futurista. Il Manifesto di Corra e Settimelli (1914). Il Manifesto inedito di Carmelich e Dolfi (1923). Il Manifesto di F T. Marinetti, “misuratore di commedie" (1927) ", Avanguardia, XV, n. 44, Roma, 2010, p. 41-59.

Marinetti parle de "misurazioni » pour désigner son activité de critique théâtral. Par « commemorazioni in avanti » il faut entendre ses discours et ses conférences sur les grands auteurs de la littérature italienne: cf. Matteo D'Ambrosio, Le commemorazioni in avanti di F. T. Marinetti. op. cit. [NDLT].

47. "vecchio problema letterario filosofico "; "verità storica »; " non essendo raggiungibile in modo assoluto è da scartarsi »; "verità molto relativa »; « orgoglio patriottistico »; «storia romanzata »; « prestigio di Roma».

48. "Consideriamo come indispensabile per la spiritualità italiana fascista mussoliniana la traduzione e pubblicazione in Italia dei capolavori autentici di tutte le letterature straniere. Consideriamo altresì indispensabile per l'autarchia letteraria ora urgente scartare dalla traduzione e pubblicazione i tre quarti delle opere straniere che alcuni editori impongono basandosi sul non abbastanza vituperato antico $e$ permanente vizio italiano che noi chiamiamo esterofilia. Questa esterofilia avendo come sua conseguenza la denigrazione del prodotto letterario italiano trova nella moltiplicazione di mediocrissimi romanzi il suo ignobile alimento. Quindi proponiamo che la traduzione e pubblicazione di opere straniere sia a priori 
diminuita di metà e rigorosamente disciplinata. Siamo pronti a dimostrare libro alla mano la vecchia e ammorbante stupidità pessimista e le minutissime cretinerie disfattiste o antitaliane che caratterizzano libri stranieri come Le stelle stanno a guardare », F. T. Marinetti, Corrado Govoni, «Autarchia letteraria", Autori e Scrittori, Roma, II, n.12, décembre 1937, p. 8. Nous transcrivons ici entièrement le manifeste, ce qui explique l'absence d'une annexe 5.

49. F. T. Marinetti, «Contro l'esterofilia. Manifesto futurista alle signore e agli Intellettuali », Gazzetta del Popolo, Torino, 24 septembre 1931, p. 3.

50. "massimo poeta della Francia contemporanea", F. T. Marinetti, "Un Poeta guarda la Croce", Gazzetta del Popolo, Torino, 8 octobre 1938, p. 3. Cf. la lettre de Claudel publiée quelques jours plus tard (le 14 octobre) par le journal quotidien romain Il Tevere.

51. Cf. les nombreux articles sur le sujet, publiés dans Autori e Scrittori en 1937.

52. F. T. Marinetti, «L'Autotreno del libro », Gazzetta del Popolo, Torino, 24 août 1932, p. 3.

53. «il problema della bonifica libraria »; «Relazione sull'attività del primo semestre 1938 », Autori e Scrittori, Roma, III, n. 7, juillet 1938, p. 13.

54. La Gazzetta del Popolo, Torino, 28 décembre 1930, p. 3.

55. Roma, Edizioni dell'Autore, 1940.

56. "Il Primo problema autarchico è l'italianità della lingua. [...] Deve smettersi l'uso di parole straniere [...] vogliamo togliere dalla lingua italiana tutte le parole straniere [...]», F. T. Marinetti Accademico d'Italia, Prefazione à Adelmo Cicogna, Autarchia della lingua, Roma, Edizioni dell'Autore, 1940, p. 7 et 8. C'est Marinetti qui souligne.

57. Domenico Tumiati, Nell'Africa Romana. Tripolitania, Milano, Treves, 1905.

58. «Si distinguono in letteratura le opere di Ferdinando Martini (Africa Italiana) Edmondo De Amicis ( Marocco) F. T. Marinetti (Mafarka il futurista - Gli Indomabili - Il Tamburo di fuoco - Il fascino dell'Egitto - Il Poema [africano] della Divisione 28 Ottobre) Domenico Tumiati (Tripolitania) Guelfo Civinini (Abissinia) Appelius Milanesi Bruno Corra Victor Hugo Gerard de Nerval Baudelaire Flaubert Loti Jean Lorrain André Gide Paul Morand Randau », F. T. Marinetti, "L'Africa generatrice e ispiratrice di Poesia e Arti ", Autori e Scrittori, Roma, III, n. 10, octobre 1938, pp. 3-4 (avec le sous-titres Relazione di F. T. Marinetti all'ottavo Convegno Volta). Nouvelle version avec beaucoup de variantes, surtout dans les deux premiers paragraphes, dans le « Manifesto Poesia plastica musica e architettura africane ", du 1934.

59. I Dieci, Lo zar non è morto. Grande romanzo d'avventure, Roma, Edizioni dei Dieci - Sapientia, 1929.

60. Cf. la sua polemica lettera aperta " Al Presidente dei "Dieci" ", L'Impero, Roma, 8 juin 1928.

61. Cf. F. T. Marinetti, « Formula letteraria artistica del Futurismo nel suo Trentennale », A. L. A., Roma, IX, n. 57, 9 février 1939.

62. Cette fois orthographié « Dujardins ».

63. «Romanzone analitico social-pessimista comunisteggiante (Thomas Mann Jules Romains) degenerazione del "monologo interiore" di Dujardin dove Proust e Joyce diluiscono le nostre parole in libertà sintetiche dinamiche simultanee in una sciolta di parole», F. T. Marinetti - Sansepolcrista Volontario della guerra africana, Pino Masnata - Volontario della guerra africana, Enrico Masnata - Volontario della guerra di Spagna, Domenico Belli - Volontario della guerra africana, Mario Menin - Volontario della guerra africana, Bruno Aschieri - Volontario della guerra di Spagna, Mino Somenzi - Granatiere di Ronchi Legionario Fiumano, Gaetano Pattarozzi - $2^{\circ}$ Littore della Letteratura, «Manifesto degli aeropoeti nel trentennale del futurismo», Autori e Scrittori, Roma, IV, n. 9 , septembre 1939 , p. 8.

64. " assoluta sincerità alogica della coscienza e del sub-cosciente "; " alcune [...] in special maniera come Dunes, 1911 contengono anche il cosiddetto "monologo interiore" ». Cf. F. T. Marinetti, "Joice [sic] e le parole in libertà », Sant'Elia, Roma, II, n. 4, 15 février 1934, p. 10.

65. F. T. Marinetti, « Dune », Lacerba, Firenze, II, n. 4, 15 février 1914, p. 51-53. 
66. La version française sera proposée à nouveau dans Les Mots en liberté futuristes, Milano, Edizioni futuriste di « Poesia », 1919, p. 85-83.

67. F. T. Marinetti, « Manifesto tecnico della letteratura futurista», Milano, 11 mai 1912 ; Milano, Direzione del Movimento Futurista. Tract.

68. A. L. A., Roma, IX, n. 110, 24 mais 1939.

69. Cette fois orthographié « Duyardin ».

70. « Romanzone analitico socialpessimista comunisteggiante (Thomas Mann Jules Romains) tipo moderato del monologo interiore di Dujardin che Proust e Joyce corrompendo le nostre parole in libertà essenziali simultanee in tutte le parole vane portarono un vomito psicologico a ripetizione. »

71. F. T. Marinetti Accademico d'Italia Luigi Scrivo Piero Bellanova, «Il romanzo sintetico. Manifesto futurista ", Il Giornale d'Italia, Roma, 24 décembre 1939, p. 3. Par la suite le manifeste paraîtra à nouveau, signé seulement par Marinetti, par exemple dans L'Ora della sera, Palermo, 17 janvier 1940, p. 3 ; les 11 points conclusifs seront publiés dans un article intitulé « Marinetti illustrerà $a$ Palermo il manifesto futurista per il "romanzo sintetico" ".

72. Piero Bellanova, Picchiata nell'amore, prefazione di F. T. Marinetti, presentazione di Luigi Scrivo, Roma, Unione Editoriale d'Italia, 1940.

73. "aspetto anacronistico, inutile e roboante [...] sottolinea soprattutto la discrepanza tra le pretese rivoluzionarie e la realtà conservatrice, accentuando il sapore di un ritorno all'ordine e alla tradizione ", Silvia Contarini, «Il Futurismo e il romanzo. Sam Dunn è morto, Il codice di Perelà », Narrativa, Paris, n. 9,1996, p. 18.

74. « un genere marginale nella produzione letteraria del movimento », id., p. 21.

75. «nessun entusiasmo per il romanzo, e scarsissimo interesse per un genere che considerano superato $e$ invecchiato [...] obsoleto e privo di interesse ", ibidem.

76. Id., p. 17.

77. Cf. Barbara Meazzi, « Seduzioni d'avanguardia, in L'arte futurista di piacere - Sintesi di tecniche di seduzione ", Barbara Meazzi (éd.), Cuneo, Nerosubianco Edizioni, collana Le Drizze, 2011, p. 127-139.

78. Le 11 octobre 1939, Marinetti avait prononcé à Acitrezza une conférence sur Verga (cf. Matteo D’Ambrosio, Le “Commemorazioni in avanti" di F. T. Marinetti, op. cit., p. 157-166). Sur la présence des livres du premier Marinetti dans la bibliothèque de l'écrivain sicilien $c f$. l'article de Rodolfo De Mattei « Marinetti inviava i libri all'amico Giovanni Verga », Gazzetta dell'Emilia, Bologna, 15 juin 1950. 79. «le forme verbali futuriste [...] sembrano incompatibili con le esigenze del romanzo », S. Contarini, op. cit., p. 19.

80. F. T. Marinetti, «Dalle parole in libertà nasce il nuovo stile narrativo ", Cronache d'attualità, Roma, V, gennaio 1921, p. 58-60 (dans la rubrique « Cronache futuriste »).

81. "nasce dal paroliberismo », F. T. Marinetti, « Lo stile parolibero », in Gli Indomabili, Piacenza, Edizioni futuriste di «Poesia» della Società Tipografica Editoriale V. Porta, 1922; nouvelle édition : Milano, Luigi Ballerini (éd.), Oscar Mondadori, 2000, p. 3.

82. Ensuite en ouverture à $F$. T. Marinetti presenta i Nuovi poeti futuristi, Roma, Edizioni di «poesia » , 1925, p. 5-19; Spagna veloce e toro futurista. Poema parolibero seguito dalla teoria delle parole in libertà, Milano, G. Morreale, 1931 (encore intitulé Lo stile parolibero).

83. À commencer par celui souvent cité de Dominique Braga.

84. Le titre n'est même pas indiqué correctement.

85. L'esthétique futuriste est notoirement dépourvue de la notion de simulation, ce qui comporterait la reconnaissance du fait que le continuum du système linguistique verbal est tout simplement bloqué. Cela entraînerait une redéfinition du principe de simultanéité et, dans les arts visuels, de la phénoménologie de toutes les formes de mouvement, simulée simplement par le signe pictural déposé sur un support statique bidimensionnel.

86. F. T. Marinetti, Gli Indomabili, op. cit. 
87. F. T. Marinetti, Originalità russa di masse distanze radiocuori [1942], Maria Delfina Gandolfo (éd.), Michele Colucci (préf.), Roma, Edizioni Voland, 1996.

88. F. T. Marinetti, Venezianella e Studentaccio [1943-44], Patrizio Ceccagnoli e Paolo Valesio (éd.), Milano, Oscar Mondadori, 2013.

89. Cf. «Qualità e difetti della letteratura d'oggi. La conferenza Marinetti al Rossetti », Piccolo della sera, Trieste, 2 décembre 1929, p. II.

90. Comme par exemple le compte rendu de Retroscena de Mario Carli publié dans la Gazzetta del Popolo le 21 février 1931.

91. Bruno Corra, Sam Dunn è morto. Romanzo futurista, Milano, Edizioni futuriste di "Poesia ", 1917.

92. Cf. par exemple Salvatore Deledda, "Per una grande arte fascista ", Mediterraneo futurista, Cagliari, II, n. 3, juin 1938, p. 3.

93. Un futuriste inconnu de Marinetti, un certain Forfanello, déclara que son idée de "romandisque " avait dépassé les propositions contenues dans le Manifeste. Questionné à ce propos, Marinetti, malade, répondit en faisant écouter son 72 tours Amanti simultanei de 1932. Cf. Il Lavoro fascista, Roma, 4 avril 1940 (avec une interview de Marinetti sur le "roman synthétique » et Amanti simultanei) et "Intervista con Marinetti sul "romanzo sintetico" » (Il Popolo di Trieste, 12 avril 1940).

94. Cf. F. T. Marinetti, " "Gli Adoratori della Patria" di Geppo Tedeschi e la teoria polemica del romanzo sintetico », Autori e Scrittori, Roma, VI, n. 10, octobre 1941, p. 1-5.

95. La lettre est conservée à la Beinecke Rare Book \& Manuscript Library, Yale University.

96. F. T. Marinetti, L'Aeropoema di Gesù [1944], Montepulciano, Editori del Grifo, s.d. mais 1991.

97. Cesare Angelini, Invito in Terrasanta, Pavia, Ancora, 1937.

98. F. T. Marinetti Sansepolcrista Accademico d'Italia, « Padre Cesare Angelini massimo scrittore cattolico secondo il pensiero del poeta Marinetti », Autori e Scrittori, Roma, VI, n. 5, mai 1941, p. 1. La lettre est conservée à la Beinecke Rare Book \& Manuscript Library, Yale University. F. T. Marinetti, L'Aeropoema di Gesù [1944], op. cit.

99. F. T. Marinetti, «Il Tattilismo. Manifesto futurista », Milano, Direzione del movimento futurista, 11 janvier 1921.

100. Cf. Svaticanamento. Dichiarazione agli Italiani di Emilio Settimelli Ottone Rosai Remo Chiti Alberto Maurizio Bruno Rosai, Firenze, Edizioni Fiorentine », s.d. mais 1931.

\section{RÉSUMÉS}

Dans la période considérée, marquée par de profonds changements dans la politique du régime fasciste (« africanisme » colonial, proclamation de l'Empire, autarchie, approche de la Deuxième Guerre mondiale), Marinetti ne s'occupe pas exclusivement et frénétiquement de la diffusion du mouvement futuriste. Dans les manifestes que nous proposons ici et qui sont reliés à ses activités d'Académicien, il ressort qu'entre la politique du régime et les programmes du mouvement il y a un lien très fort. Pendant ce temps, le renouvellement des arts, pourtant incessamment annoncé, n'est de fait inexistant, tant au niveau des intentions exprimées que dans les résultats obtenus.

Nel periodo considerato, segnato da profonde trasformazioni nella politica del regime fascista ("africanismo" coloniale, proclamazione dell'Impero, autarchia, avvicinamento al secondo conflitto mondiale), Marinetti non si occupa solo e freneticamente delle fortune del movimento 
futurista. I manifesti riproposti, da collegare alla sua attività di Accademico, caratterizzata da numerose responsabilità istituzionali, evidenziano l'inseparabilità delle argomentazioni riguardanti la politica del regime dai programmi del movimento, impegnato in una propria modellizzazione delle pratiche discorsive della propaganda. Intanto il rinnovamento nelle arti, che si vorrebbe continuare a garantire, non è riconoscibile né negli intendimenti espressi né nei risultati ottenuti.

\section{INDEX}

Index géographique : Italie

Index chronologique : 1936-1939

Mots-clés : futurisme, fascisme, poésie futuriste, roman futuriste, Empire d'Italie, colonialisme

\section{AUTEUR}

\section{MATTEO D'AMBROSIO}

Matteo D'Ambrosio a été professeur d'histoire de la critique littéraire à l'Université de Naples Federico II (1991-2011). Fulbright Fellow à la Harvard University, il a étudié à la Yale University et au Getty Research Institute de Los Angeles. Il est l'auteur de nombreuses publications consacrées à la recherche artistique et littéraire des avant-gardes : futurisme, dadaïsme, surréalisme, lettrisme, poésie concrète, poésie visuelle, poésie phonétique et sonore, vidéo poésie, littérature informatique. Il est membre du Comité scientifique pour les célébrations du 50 e anniversaire de la mort de F. T. Marinetti (1994-96) et du Comité scientifique pour les célébrations du centenaire du premier manifeste futuriste (2009). 